\title{
Development of an advanced noise reduction method for vibration analysis based on singular value decomposition
}

\author{
Wen-Xian Yang ${ }^{\mathrm{a}, \mathrm{b}, *}$, Peter W. Tse \\ ${ }^{a}$ School of Engineering, Nottingham Trent University, Nottingham, NG1 4BU, UK \\ ${ }^{\mathrm{b}}$ Institute of Vibration Engineering, Northwestern Polytechnical University, Xi'an 710072, People's Republic of China \\ ${ }^{\mathrm{c}}$ Department of Manufacturing Engineering \& Engineering Management, City University of Hong Kong, Hong Kong, People's Republic of China
}

Received 31 January 2003; revised 16 March 2003; accepted 17 March 2003

\begin{abstract}
The paper developed a reasonable and practical method for identifying the useful information from the signal that has been contaminated by noise, so that to provide a feasible tool for vibration analysis. A new concept namely the Singular Entropy (SE) was proposed based on the singular value decomposition technique. With the aid of the SE, a series of investigations were done for discovering the distribution characteristics of noise contaminated and pure signals, and consequently an advanced noise reduction method was developed. The experiments showed that the proposed method was not only applied for dealing with the stationary signals but also applied for dealing with the non-stationary signals.
\end{abstract}

(C) 2003 Elsevier Science Ltd. All rights reserved.

Keywords: Singular value decomposition; Singular entropy; Noise reduction

\section{Introduction}

Affected by the violent background vibration or the strong environmental electromagnetic disturbances, the signals collected from field are often inevitably contaminated by noise. In order to reduce the noise residents in the signals, many methods had been tried, such as time series analysis [1,2], wavelet analysis [3-7], parameter estimation algorithms [8-12], special numerical filters [13-21] and so on. However, the long-term practice proves that these methods have their own limitations more or less. For example, in order to set up an appropriate mathematical model, not only a large scale of data is required, but also an intensive calculation is often involved in time series analysis. As regard to the wavelet-based de-noising techniques, the selection of wavelet function is crucial for the noise reduction effect. However, up to date, because of the complexity of the real signals in composition, how to select a suitable wavelet function for a special signal is still a knotty problem expected to solve. Parameter estimation

\footnotetext{
* Corresponding author. Present address: School of Engineering, Nottingham Trent University, Nottingham NG1 4BU, UK.

E-mail address: wen.yang@ntu.ac.uk (W.-X. Yang).
}

algorithms often work well when the value of the Signal to Noise Ratio (SNR) of the inspected signal is high, while it is not competitive any longer when the value of the SNR is low. Due to the influence of noise, it is often difficult to estimate the frequency distribution of a noised signal. This limits the application of numerical filters to a great extent. Additionally, some other approaches have also been tried in recent years [22-26]. Among them, the singular value decomposition (SVD) based de-noising method is recognised as a competitive one for its simple operation and less calculation.

The SVD technique has been widely used in many fields in recent years, such as acoustics [27], smart control [28,29], electronics [30,31], signal processing [22,23,32,33], mathematics [34,35] and so on. However, compared to its achievements in above-mentioned fields, the research in noise reduction field has not been done sufficiently. In order to contribute in this field, a new concept namely the Singular Entropy (SE) is proposed in this paper. After investigating the relationship between the SNR of the signal and its SE, an advanced SVD-based noise reduction method was developed consequently. Its effectiveness on noise reduction will be demonstrated by a series of experiments shown in following figures. 


\section{Singular entropy}

According to the theorem of the SVD, for a real matrix $\mathbf{A}$ with the size of $m \times n$, it always satisfies

$\mathbf{A}_{m \times n}=\mathbf{U}_{m \times l} \boldsymbol{\Lambda}_{l \times l} \mathbf{V}_{n \times l}^{\mathrm{T}}$

where the matrix $\boldsymbol{\Lambda}$ is a diagonal matrix and has the size of $l \times l$. All its diagonal elements $\lambda_{i}(i=1,2, \ldots, l)$ are nonnegative and arranged in descending order, i.e. $\lambda_{1} \geq \lambda_{2} \geq$ $\cdots \geq \lambda_{l} \geq 0$. These diagonal elements are called the singular values of the matrix $\mathbf{A}$. The practice shows that, the higher the SNR of the inspected signal, the more the number of zero valued diagonal elements will appear in $\boldsymbol{\Lambda}$. This phenomenon reveals the relationship between the matrix $\boldsymbol{\Lambda}$ and the SNR of the signal. By investigating the number of zero valued diagonal elements in $\Lambda$, the SNR of the signal can then be estimated approximately. Based on this idea, the SVD-based de-noising technique was developed as in Ref. [22]. Firstly, substitute zero for those diagonal elements with small values in $\boldsymbol{\Lambda}$, so that a new matrix $\Lambda^{\prime}$; is obtained. Secondly, replace the matrix $\Lambda$ with the new matrix $\Lambda^{\prime}$ and re-calculate the Eq. (1). In consequence, another real matrix $\mathbf{A}^{\prime}$ will be resulted. Finally, deduce the noise-reduced signal from the matrix
$\mathbf{A}^{\prime}$. Unfortunately, a knotty problem still exists in this technique and impedes the technique to be effectively used in practice. The problem is how can we judge which part of diagonal elements in the matrix $\boldsymbol{\Lambda}$ should be regarded as those with small values and replaced by zero. The practice shows that the reasonable solution for this problem is extremely crucial for the effect of noise cancellation. To ease the comprehension of this problem, the following simulated signal is considered

$x(t)=x_{0}(t)+\sigma e(t)$

where $x_{0}(t)=\sin \left(2 \pi f_{1} t+\alpha\right)+\sin \left(2 \pi f_{2} t+\beta\right), f_{1}=50 \mathrm{~Hz}$, $f_{2}=100 \mathrm{~Hz}, \alpha=\beta=0^{\circ}, e(t)$ represents the white noise, $\sigma=5$ indicates the noise level. Apply above-mentioned SVD-based technique to this signal for noise cancellation. The raw signal and the noise reduction results generated under different de-noising orders are shown in Fig. 1.

It is clearly seen from Fig. 1 that, compared to the pure signal $x_{0}$, the distortions take place on the resulted waveforms when the de-noising order is selected to be a smaller value, while much noise still residents in the signal when the order is selected to be a larger one.

In order to select the de-noising order reasonably, a new concept namely the SE is designed. The equation of

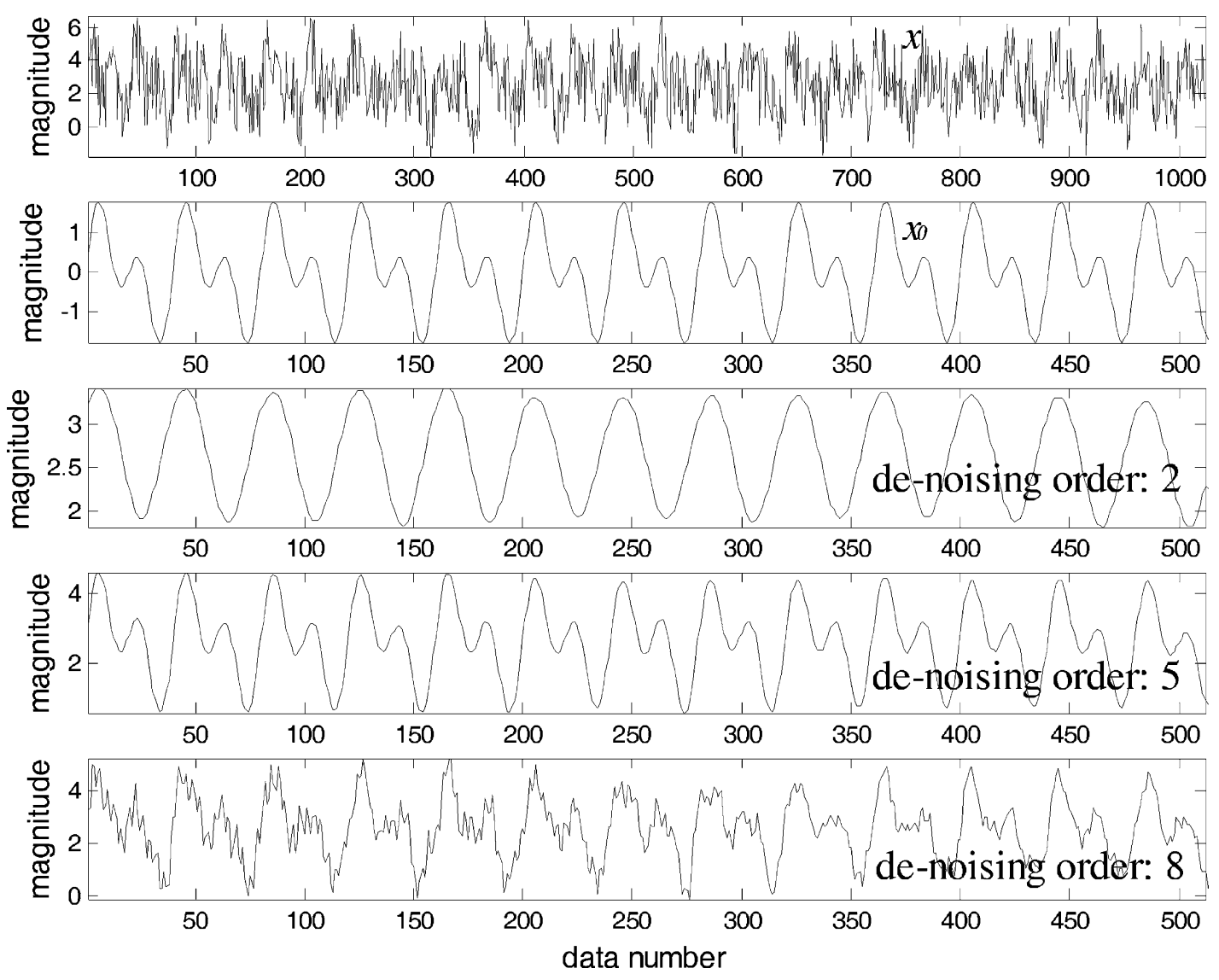

Fig. 1. Noised reduced signals obtained at different de-noising order. 
the SE is

$E_{k}=\sum_{i=1}^{k} \Delta E_{i}, \quad k \leq l$

where $E_{k}$ represents the $\mathrm{SE}$ at order $k, \Delta E_{i}$ indicates the increment of the SE at order $i$

$\Delta E_{i}=-\left(\frac{\lambda_{i}}{\sum_{j=1}^{l} \lambda_{j}}\right) \log \left(\frac{\lambda_{i}}{\sum_{j=1}^{l} \lambda_{j}}\right)$

Necessary to note that, like other types of information entropy, the SE has a similar significance on describing the information quantity of the signal, i.e. the lager the value of the SE, the more complex the signal is in composition.

\section{Selection of the de-noising order}

It has been widely recognized that the information quantity of the signal is mainly determined by the number of frequency components contained in it. In order to investigate the relationship between the SE of the signal and its information quantity, the following researches were performed.

1. The SE of the signals that contain same number of frequency components

Assume two simulated signals as

$\left\{\begin{array}{l}x(t)=A_{1} \sin \left(2 \pi f_{1} t+\alpha_{1}\right)+B_{1} \sin \left(2 \pi f_{2} t+\beta_{1}\right) \\ y(t)=A_{2} \sin \left(2 \pi f_{3} t+\alpha_{2}\right)+B_{2} \sin \left(2 \pi f_{4} t+\beta_{2}\right)\end{array}\right.$

where $A_{1}=A_{2}=B_{1}=B_{2}=1, \alpha_{1}=\alpha_{2}=\beta_{1}=\beta_{2}=$ $0^{\circ}, f_{1}=100 \mathrm{~Hz}, f_{2}=200 \mathrm{~Hz}, f_{3}=10 \mathrm{~Hz}, f_{4}=20 \mathrm{~Hz}$. Their time waveforms and the variation tendencies of their SE are shown in Fig. 2.

From the results shown in Fig. 2, it is seen that the SE curves of the signals $x$ and $y$ are completely overlapped together though the frequency components contained in them are distinctly different. This implies that the SE of the signals containing same number of frequency components will show same variation tendency. This conclusion has also been confirmed further by more simulated experiments.

2. Relationship between the saturated order of the SE and the number of frequency components contained in pure signal
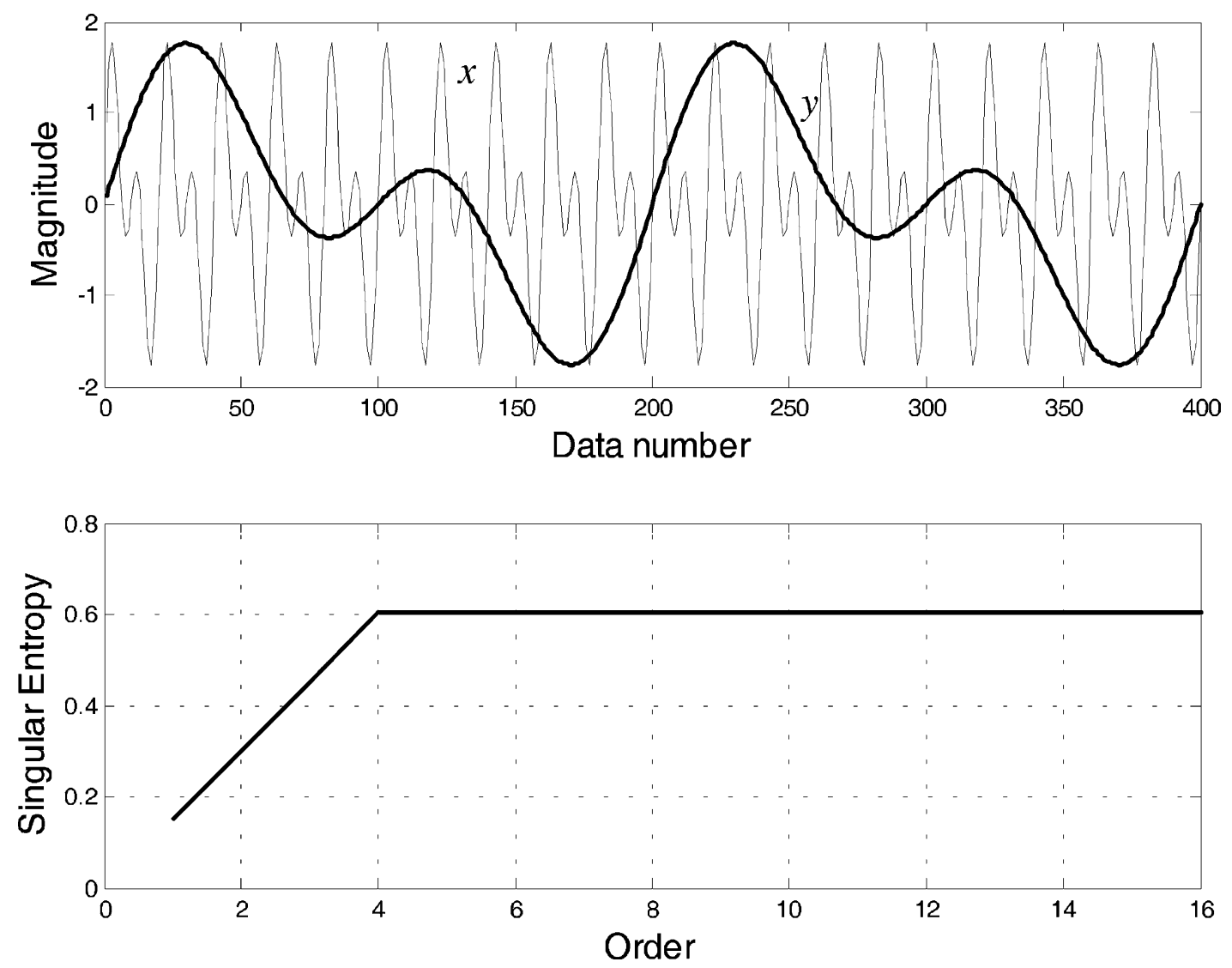

Fig. 2. Signals containing same number of frequency components. 
Given the following four simulated pure signals

$$
\left\{\begin{aligned}
x_{1}(t)= & \sin \left(2 \pi f t+\alpha_{1}\right) \\
x_{2}(t)= & \sin \left(2 \pi f t+\alpha_{1}\right)+\sin \left(4 \pi f t+\alpha_{2}\right) \\
x_{3}(t)= & \sin \left(2 \pi f t+\alpha_{1}\right)+\sin \left(4 \pi f t+\alpha_{2}\right) \\
& +\sin \left(6 \pi f t+\alpha_{3}\right) \\
x_{4}(t)= & \sin \left(2 \pi f t+\alpha_{1}\right)+\sin \left(4 \pi f t+\alpha_{2}\right) \\
& +\sin \left(6 \pi f t+\alpha_{3}\right)+\sin \left(8 \pi f t+\alpha_{4}\right)
\end{aligned}\right.
$$

where $f=10 \mathrm{~Hz}, \alpha_{1}=\alpha_{2}=\alpha_{3}=\alpha_{4}=0^{\circ}$. Their time waveforms and the variation tendencies of the SE are plotted in Fig. 3.

From Fig. 3, it can be found that the more the number of frequency components contained in the signal, the larger the saturated order of the SE is. Moreover, a definite numerical relation exists between them, i.e.

$k_{\mathrm{s}}=2 N_{\mathrm{f}}$

where $k_{\mathrm{s}}$ represents the saturated order at which the information quantity of the signal gets to a saturated state and from which the value of SE will not change any more, $N_{\mathrm{f}}$ is the number of frequency components included in the signal.

3. Relationship between the saturated order of the SE and the number of frequency components contained in noise contaminated signal

Assume a noise contaminated signal

$$
x(t)=\sin (2 \pi f t+\alpha)+\sin (4 \pi f t+\beta)+\sigma e(t)
$$

where $f=50 \mathrm{~Hz}, \alpha=\beta=0^{\circ}, e(t)$ represents the white noise and $\sigma$ indicates the noise level that is in inverse proportion to the SNR of the signal. In order to investigate the relationship between the saturated order of the $\mathrm{SE}$ and the number of frequency components contained in noised signal, the SE are calculated under a series of different values of $\sigma$. The computation results are shown in Fig. 4. For the convenience of analysis, the increment of the $\mathrm{SE}$, symbolized by $\Delta E$, is adopted in the figure.

It is found from Fig. 4 that, the saturated order $k_{\mathrm{s}}$ of the noised signal $(\sigma \neq 0)$ is nearly same as that of the pure signal $(\sigma=0)$ when the noise level $\sigma$ is very low (e.g. $\sigma=0.25,0.5$ ), while the value of $k_{\mathrm{s}}$ is one order larger than that of the corresponding pure signal when the value of $\sigma$ is high (e.g. $\sigma=1,2$ and 4), i.e.

$k_{\mathrm{s}}=2 N_{\mathrm{f}}+1$

This is because, as shown in Fig. 5, the information quantity of white noise signal may reach the saturated state at the first order.

From above experimental results, it can be easily deduced that the de-noising order should be selected to be the saturated order of the SE of the signal. Else, either the distortion of the time waveform of the signal (when the denoising order is smaller than the saturated order of SE) or the insufficient noise reduction (when the de-noising order is larger than the saturated order of SE) will take place. Based on this idea, the advanced SVD-based noise reduction technique was developed in this paper.

\section{The frequency resolution of the advanced SVD-based noise reduction method}

It is deduced from formula (7) and (9) that, the number of frequency components contained in signal dominates the saturated order of the SE. Then in return, whether the saturated order of the SE can give a correct estimate of the number of frequency components contained in signal? In order to answer this question, the following simulated experiment is performed

$x(t)=A \sin \left(2 \pi f t+\phi_{1}\right)+B \sin \left[2 \pi\left(f+\alpha f_{\mathrm{s}}\right) t+\phi_{2}\right]$

where $A=B=1, \phi_{1}=\phi_{2}=0^{\circ}, f$ is the fundamental frequency of the signal, $f_{\mathrm{s}}$ represents the sampling frequency, the coefficient $\alpha$ indicates the fluctuation of the frequency of the second component in the signal. According to Eq. (7), the saturated order of the SE of the signal $x(t)$ should be 4 . Then, take $f$ to be 50,200 and $600 \mathrm{~Hz}$, respectively. Let the signal sampling frequency $f_{\mathrm{s}}=2$ and $20 \mathrm{kHz}$, respectively. The $\Delta E$ curves of $x(t)$ under different values of $\alpha$ and $f_{\mathrm{s}}$ are calculated and plotted in Fig. 6.

From the calculation results shown in Fig. 6, it can be found that in spite of the values of the fundamental frequency $f$ and the sampling frequency $f_{\mathrm{s}}$, the saturated order of the SE of the signal $x(t)$ can be correctly estimated when $\alpha \geq 0.0009$. While when the value of $\alpha$ is smaller than 0.0009 , the identification of the saturated order of the SE tends to be difficult and a wrong evaluation may be resulted. From this, it is known that the frequency resolution of the SVD-based de-noising method is $1.8 \mathrm{~Hz}$ when the sampling frequency is $2 \mathrm{kHz}$ and $18 \mathrm{~Hz}$ when the sampling frequency is $20 \mathrm{kHz}$. Obviously, the SVD-based noise reduction method has an excellent frequency resolution, that is related to the sampling frequency of the inspected signal.

\section{Noise reduction capability of the SVD-based de-noising method}

The cares of this section are to investigate the problem of at how low value of the SNR, the SVD-based method can still distinguish the useful information from the noised signal with a satisfied noise reduction effect. In order to attain this purpose, a simulated signal is designed as follows

$x(t)=A_{1} \sin \left(2 \pi f_{1} t+\alpha\right)+A_{2} \sin \left(2 \pi f_{2} t+\beta\right)+\sigma e(t)$

where $A_{1}=A_{2}=1, f_{1}=50 \mathrm{~Hz}, f_{2}=150 \mathrm{~Hz}, e(t)$ is the white noise, $\sigma$ indicates the noise level, $\alpha=\beta=0^{\circ}$ are the original phase values of the two frequency components. According to Eq. (9), it is easily known that the saturated 

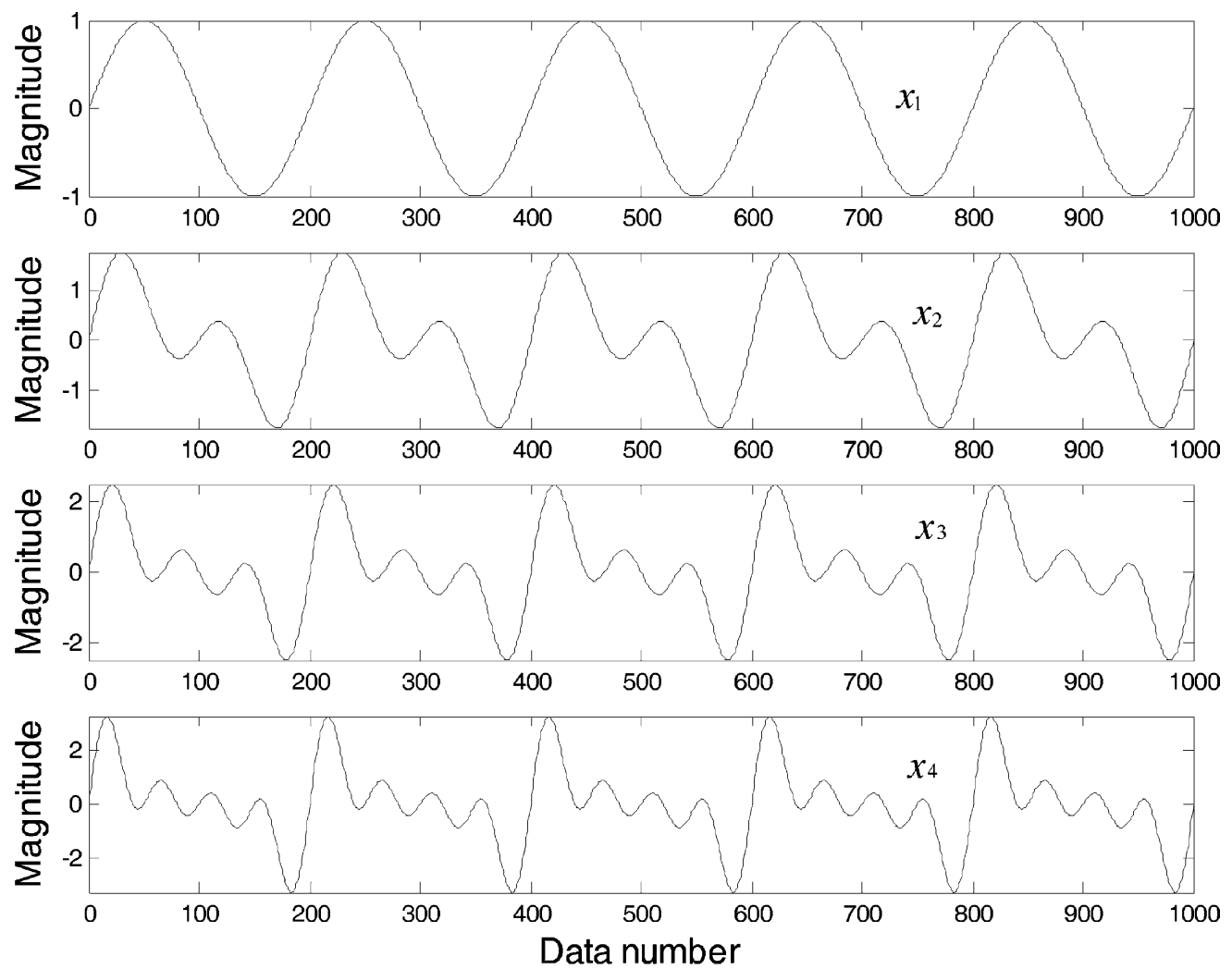

(a) time waveforms

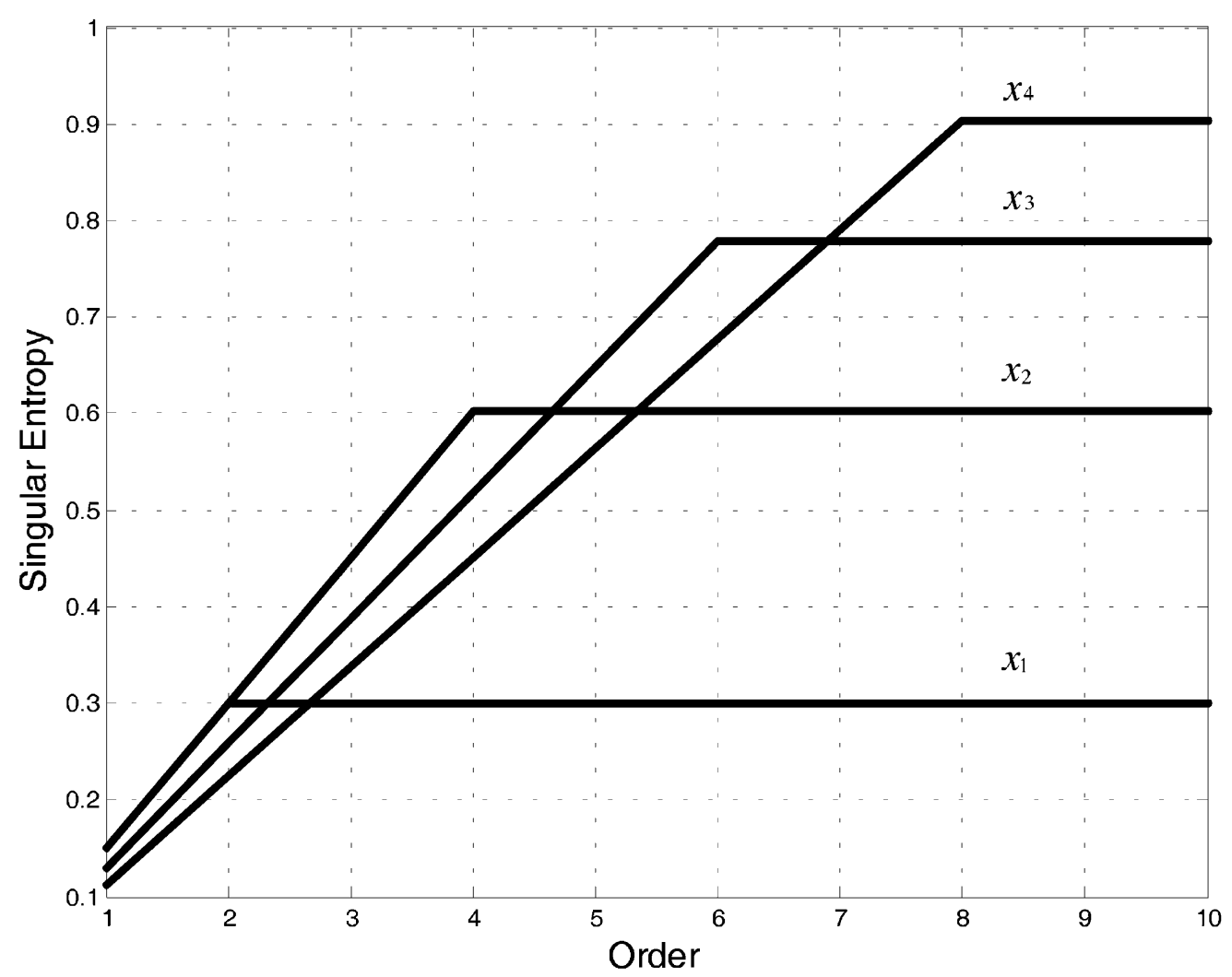

(b) variation tendencies of $\mathrm{SE}$

Fig. 3. Signals containing different number of frequency components. 

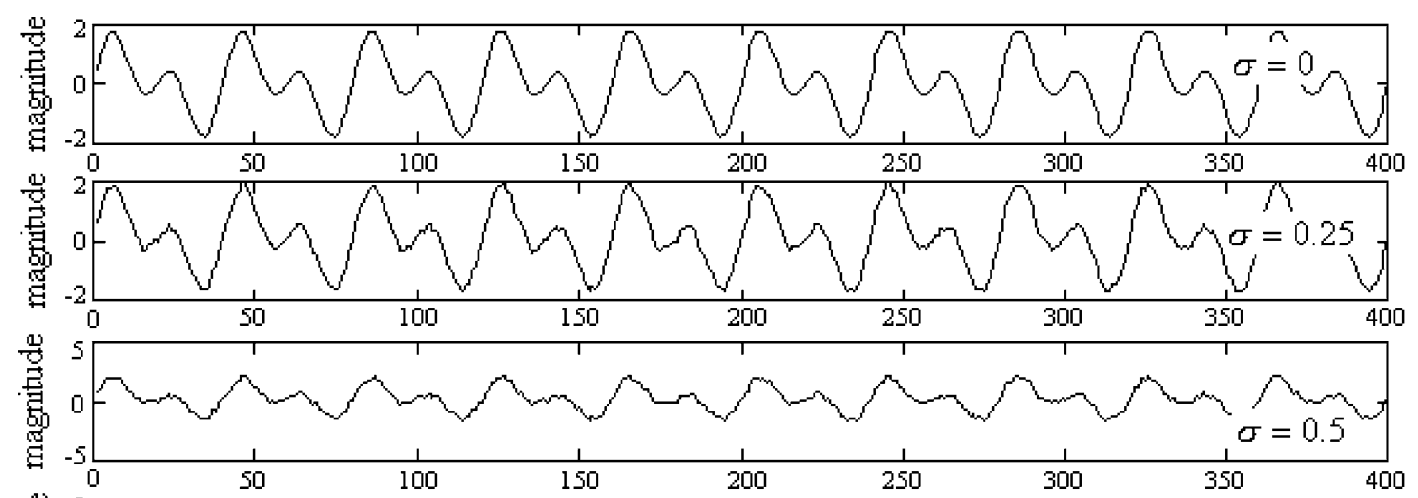

营

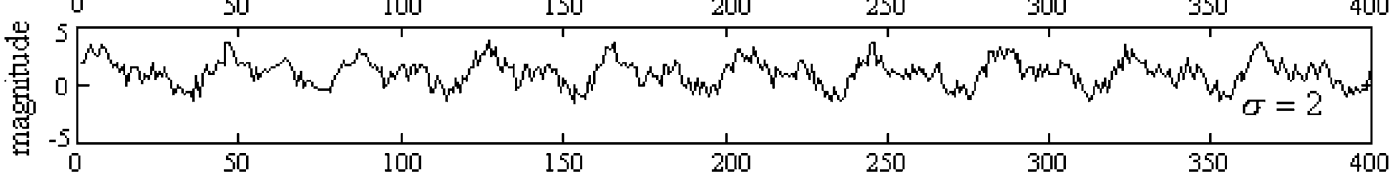

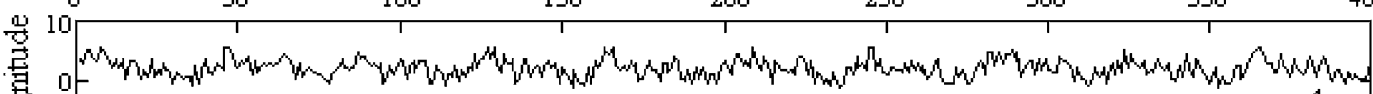

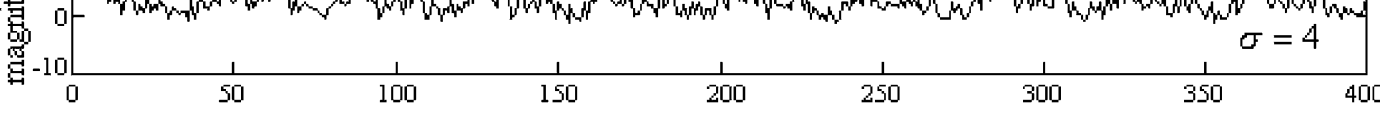
data number

(a) time waveforms of signals

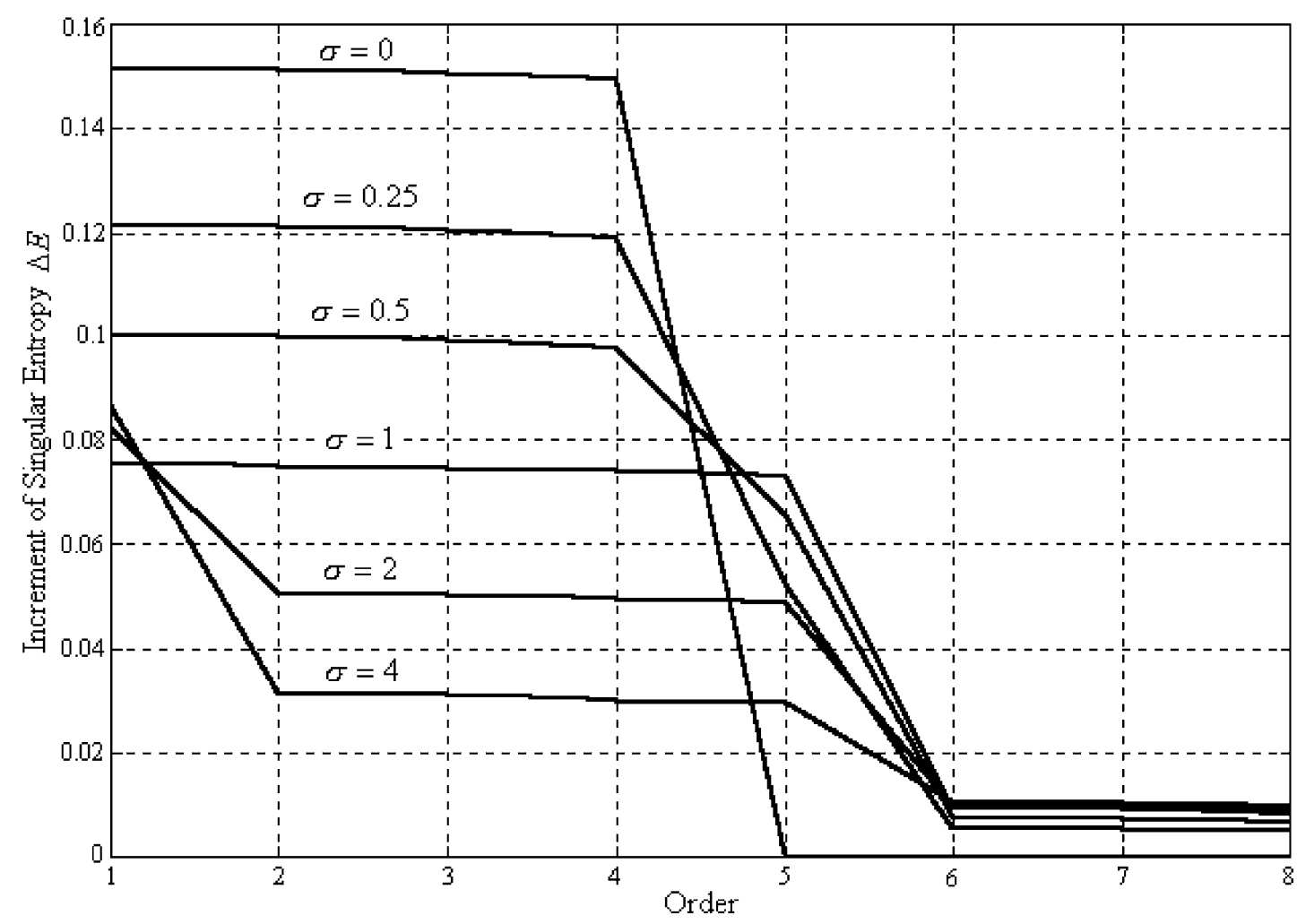

(b) the increment of SE

Fig. 4. Variation tendencies of $\Delta E$ under different values of $\sigma$. 

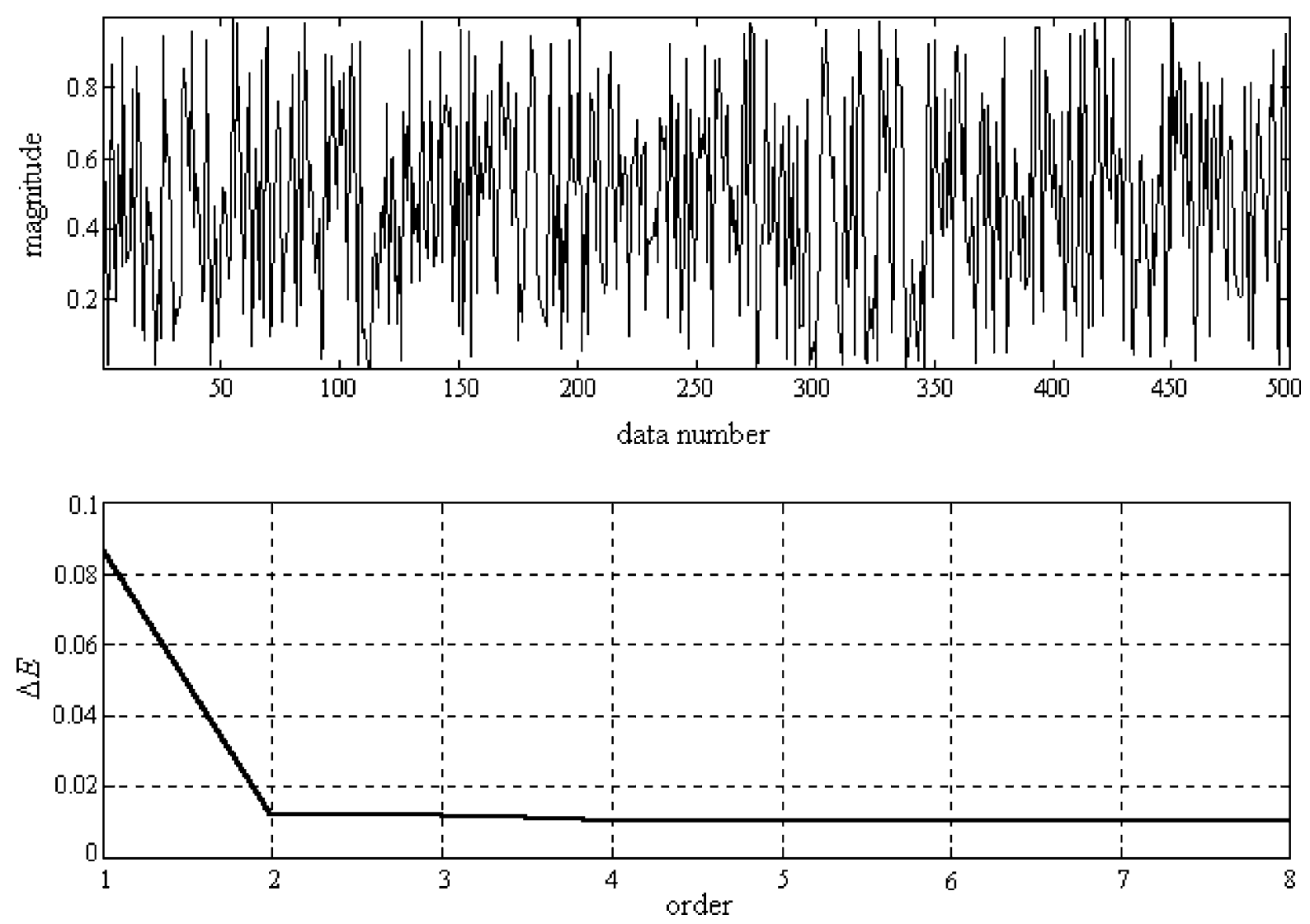

Fig. 5. The $\Delta E$ of white noise signal.

order of the SE of the signal $x(t)$ should be 5 because there are two frequency components contained in it. Let $\sigma$ be a variable, then when this saturated order cannot be correctly identified any longer from the $\mathrm{SE}$ or $\Delta E$ curve, the corresponding value of the SNR is defined as the capability limitation of the proposed method. In the following, the $\Delta E$ curves of $x(t)$ under different values of $\sigma$ are calculated and the computation results are shown in Fig. 7.

From Fig. 7, it is found that the saturated order of the SE of the signal $x(t)$ can be easily identified when $\sigma>10$. Then, the capacity limitation of the method could be roughly estimated by using the following equation

$\mathrm{SNR}_{\lim }=\frac{1}{\sigma^{2}} \sum_{i=1}^{M} A_{i}^{2}$

where $M$ indicates the number of frequency components contained in the signal. Through formulation (12), it is known that the capability limitation of the SVD-based denoising method is approximately 0.02 . Undoubtedly, such a low value of $\mathrm{SNR}_{\text {lim }}$ fully proves the strong de-noising capability of the proposed method.

\section{Experiments for noise reduction}

In order to verify the effectiveness of the proposed method in practical vibration analysis, the following experiments were performed as essential examples for further confirmations.

\subsection{Cases for stationary signal}

As the rotating machinery has a relatively stable rotating speed when it is running, the vibration signals collected from its rotor are usually regarded as stationary signals. But affected by the violent background vibration or strong electromagnetic disturbances, the signals collected from field are often contaminated by noise. Fig. 8 shows a vibration signal collected from the rotor of an air compressor served in a chemical plant, where the shaft misalignment failure takes place on the rotor system. It is clearly seen that some noises resident in the signal.

In order to reduce the noise in the signal, the $\Delta E$ of the signal is calculated and its curve is plotted in Fig. 8 too. From the curve of $\Delta E$, it is found that the information quantity of the signal reaches the saturated state at order 4 . According to the research conclusions obtained above, it can be deduced that the noise contamination is not serious and there are probably two main frequency components contained in the signal. Therefore, the optimum de-noising order of this signal should be 4 . Based on this analysis, the signal is processed and the calculation result is shown in the bottom plot of Fig. 8. From the noise reduction result, it can be concluded that with the aid of the saturated order of 

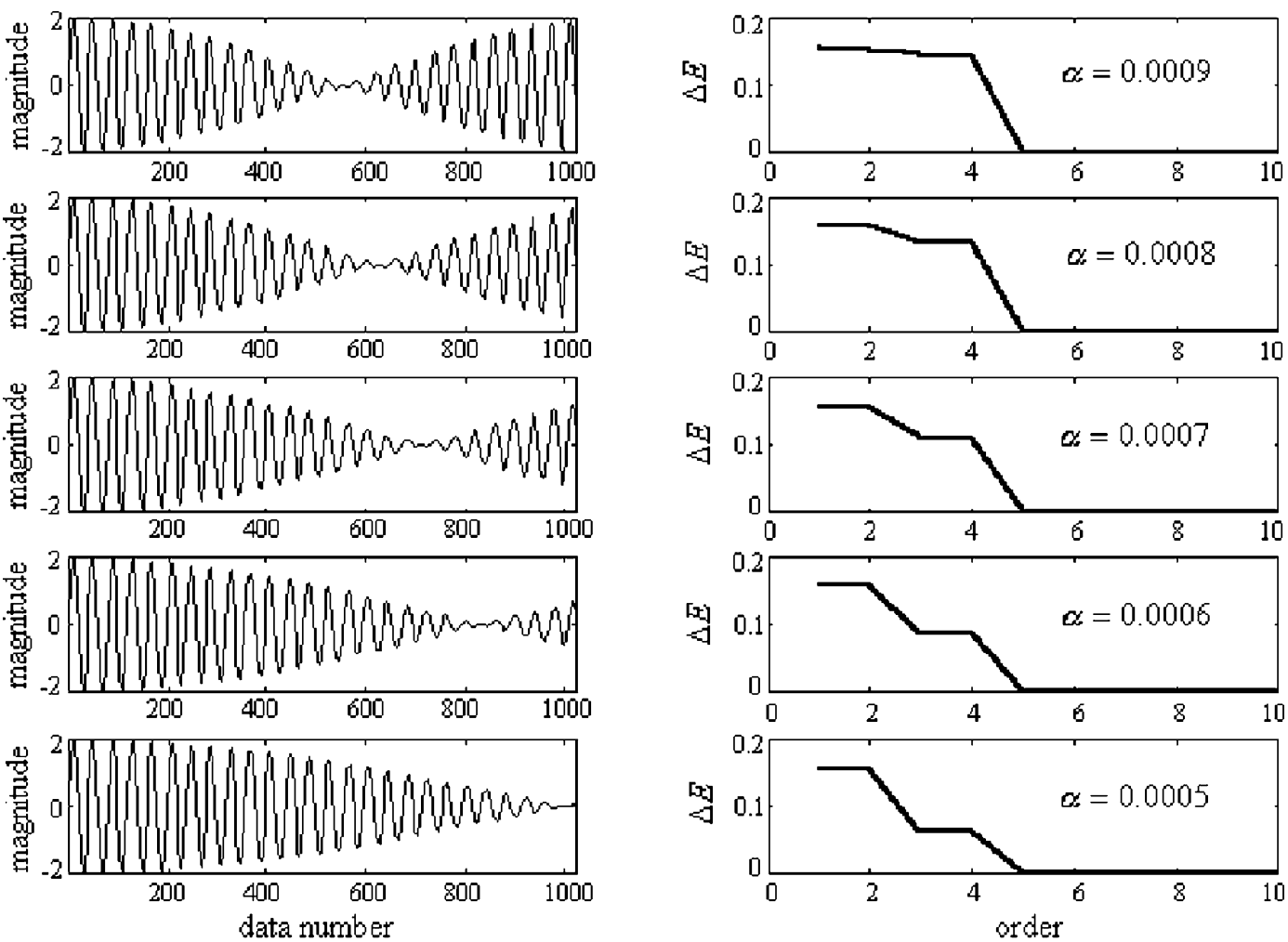

(a) $f=50 \mathrm{~Hz}$ and $f_{s}=2 \mathrm{kHz}$
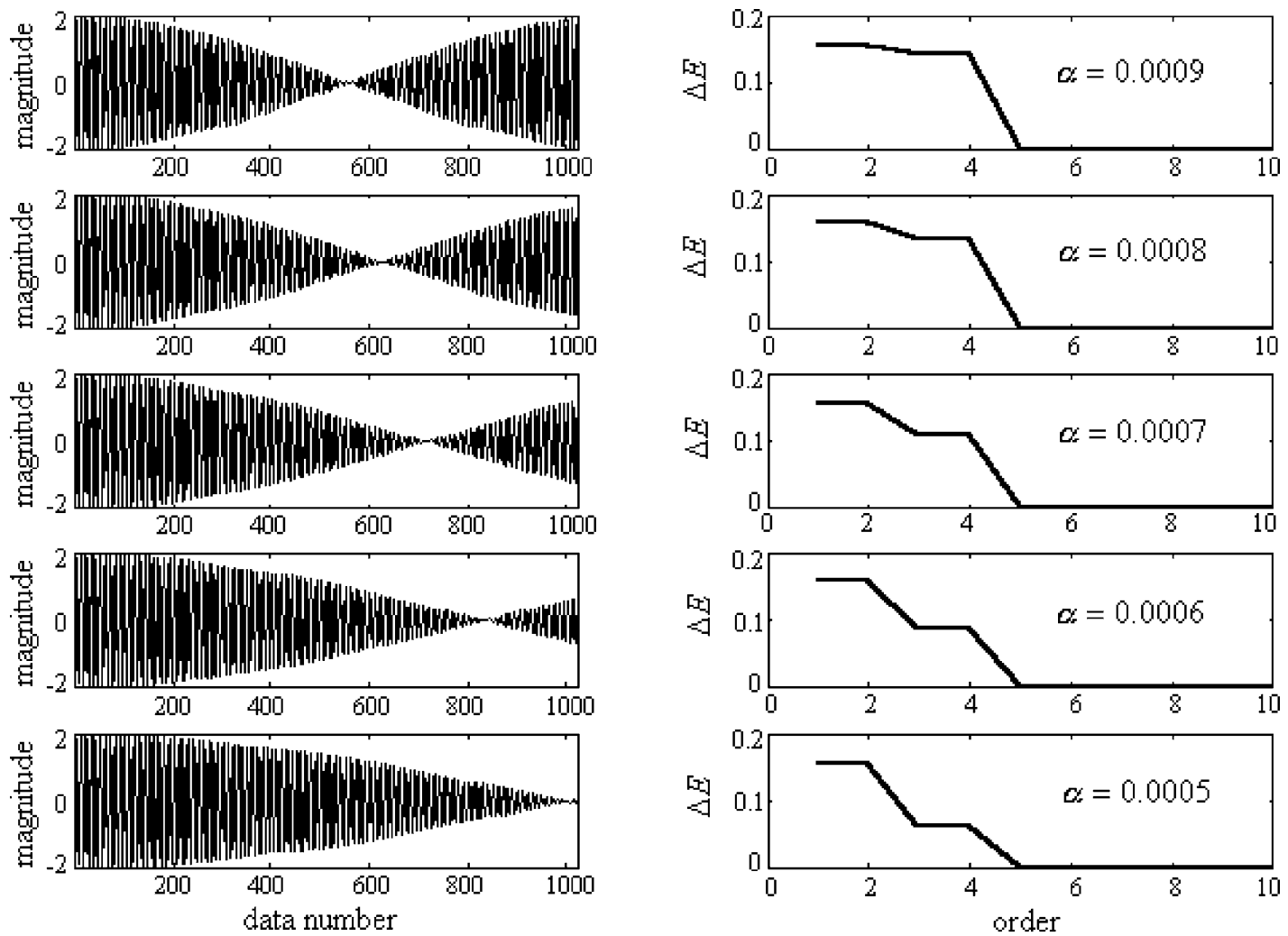

(b) $f=200 \mathrm{~Hz}$ and $f_{s}=2 \mathrm{kHz}$

Fig. 6. The $\Delta E$ at different $f$ and $f_{\mathrm{s}}$. 

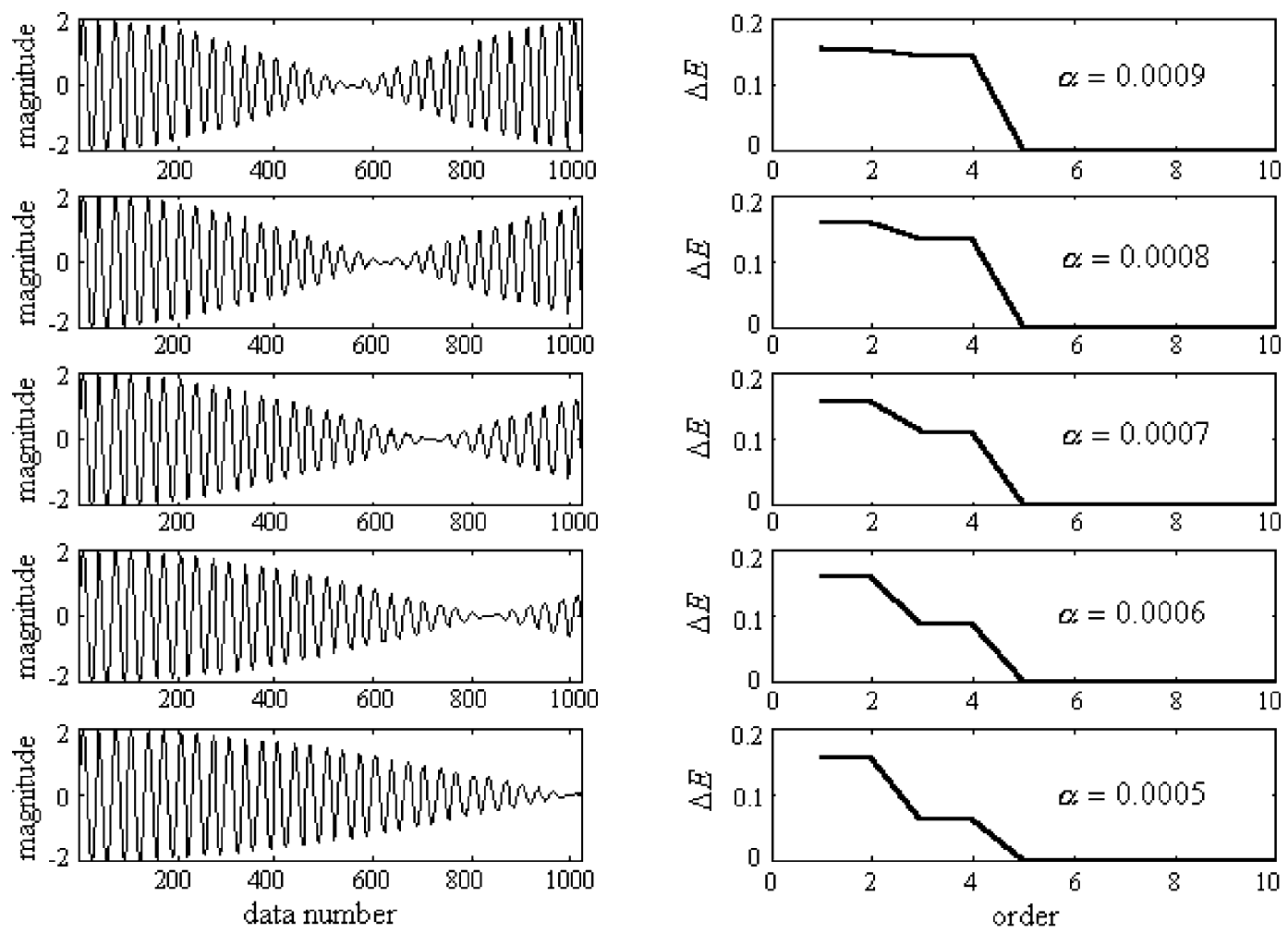

(c) $f=600 \mathrm{~Hz}$ and $f_{s}=20 \mathrm{kHz}$

Fig. 6 (continued)
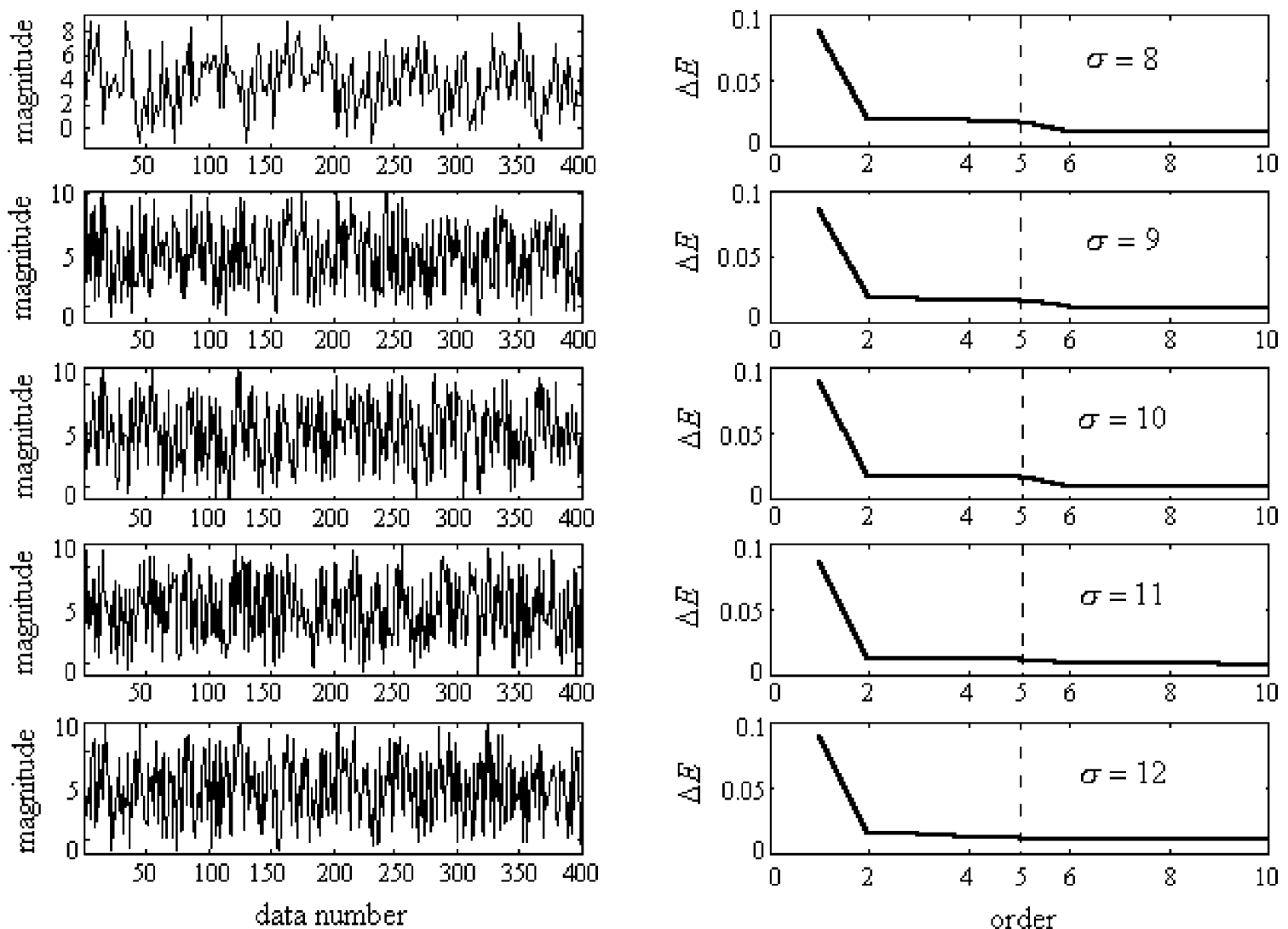

Fig. 7. The $\Delta E$ curves under different values of $\sigma$. 

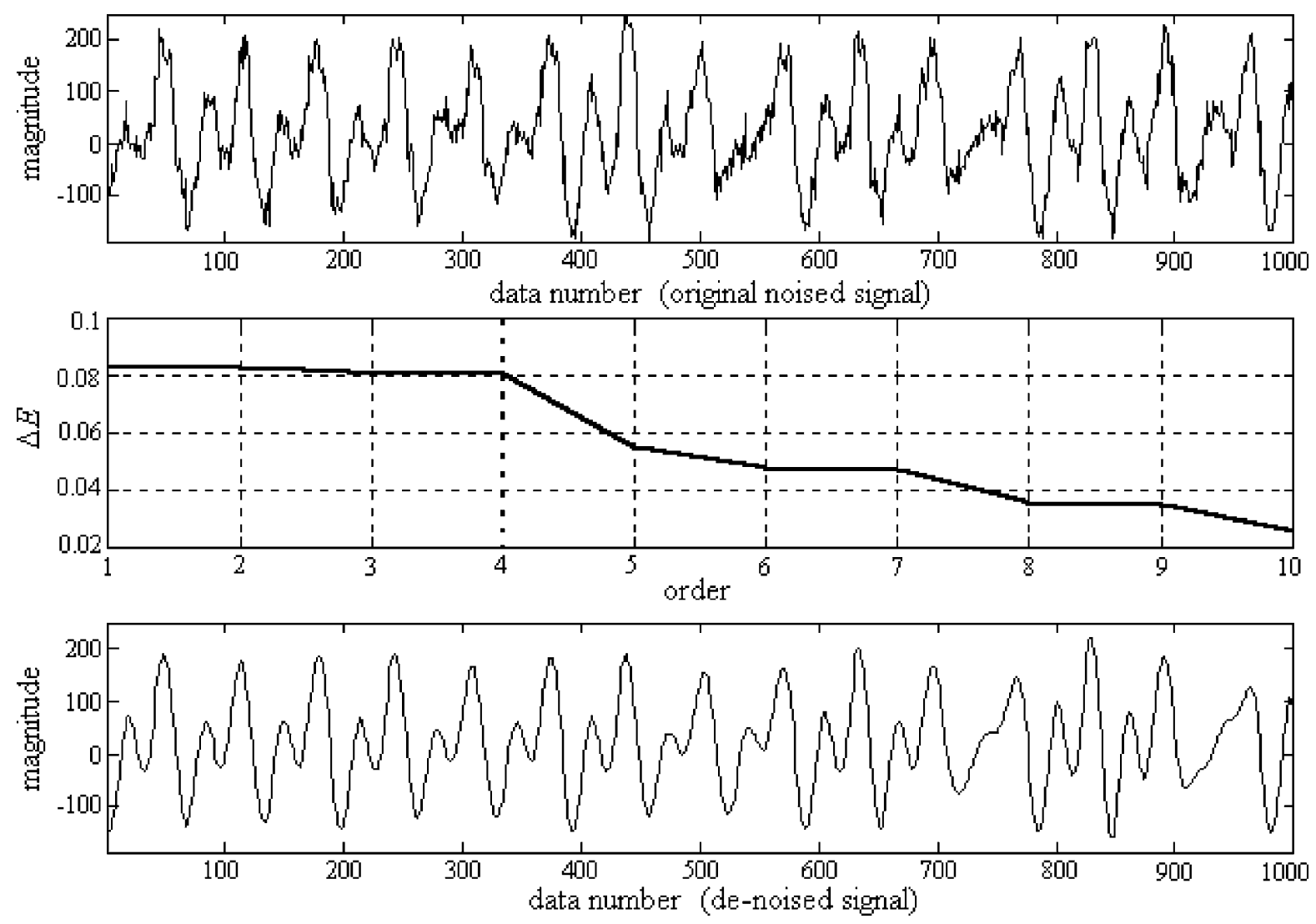

Fig. 8. Noise contaminated vibration signal collected from the rotor.

the SE, a wonderful noise reduction effect can be actually obtained.

\subsection{Cases for non-stationary signals}

Different from stationary signals, much more noise and shock signals will possibly be present in non-stationary signals. As an aid of the proposed noise reduction method, the following calculation will be further done on the noisereduced signals in this case so that the insignificant features in the signal could be depressed while the useful features sufficiently bulged.

$$
x^{\prime \prime}(t)=x^{\prime}(t)^{3} \max \left(\left|x^{\prime}(t)\right|\right) / \max \left(\left|x^{\prime}(t)^{3}\right|\right)
$$

where $x^{\prime \prime}(t)$ is the final result of the noise cancellation, $x^{\prime}(t)$ is the noise-reduced signal generated by the SVD-based denoising method. In this way, the useful information will be magnified while the noise depressed. This improvement is more suitable to be used to distinguish the impulses contained in non-stationary signals. In order to verify the effectiveness of this advanced method, the following tests were performed. Fig. 9(a) shows an acceleration signal collected from a rolling element bearing when an outer race failure happens. Obviously, the raw signal is so confused and not any useful information can be found directly from it.

After calculating the SE and its increment of this signal, it is found from Fig. 9(b) that the information quantity of the signal approaches to be saturate at order 8 , the increment of the information quantity after this order can be regarded as the effect of noise. So select the order 8 as the order for noise cancellation, the noise reduction result obtained under this order is plotted in Fig. 9(a) too. From the noise-reduced signal, the periodic impulses can be easily found though their amplitudes are different from each other. It is no doubt that this result will make the fault diagnosis be much easier than using the raw signal.

Another test was done as shown in Fig. 10, where a ball failure took place in the bearing.

Likewise, the variation tendencies of the SE and its increment $\Delta E$ are calculated and the results are plotted in Fig. 10(b). According to the $\Delta E$ curve shown in the figure, the order 19 is selected as the order for noise cancellation. The noise reduction result obtained under this order is as shown in Fig. 10(a). From the result, not only the strong impulsive features are distinguished, but also some weak impulses are also identified successfully.

From above experimental results, it is concluded that, with the aid of the SE and its increment $\Delta E$, not only the useful information contained in the stationary signals can be easily distinguished, but also the useful information contained in the non-stationary signals can be successfully identified too. 

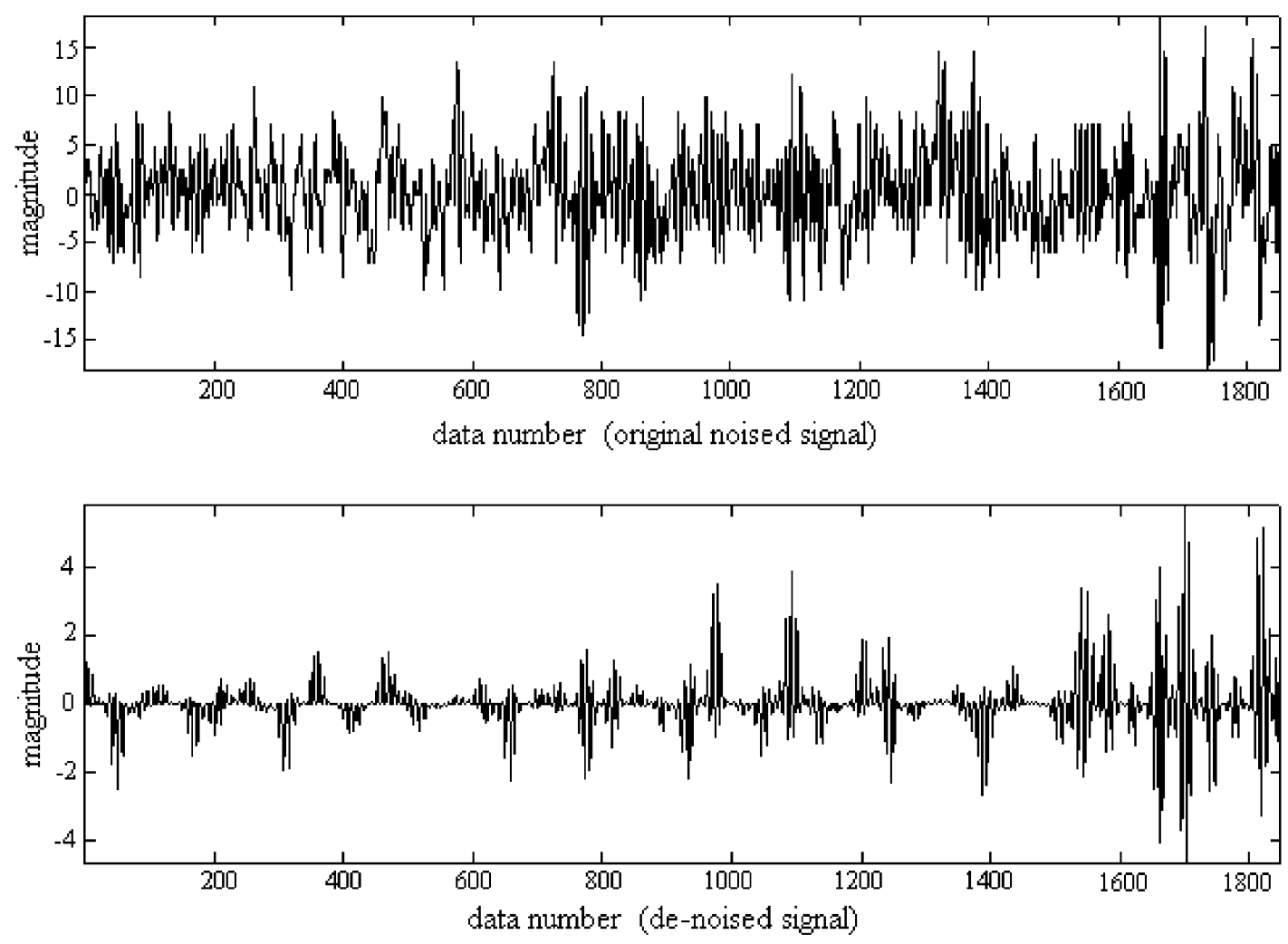

(a) raw and noise-reduced signals
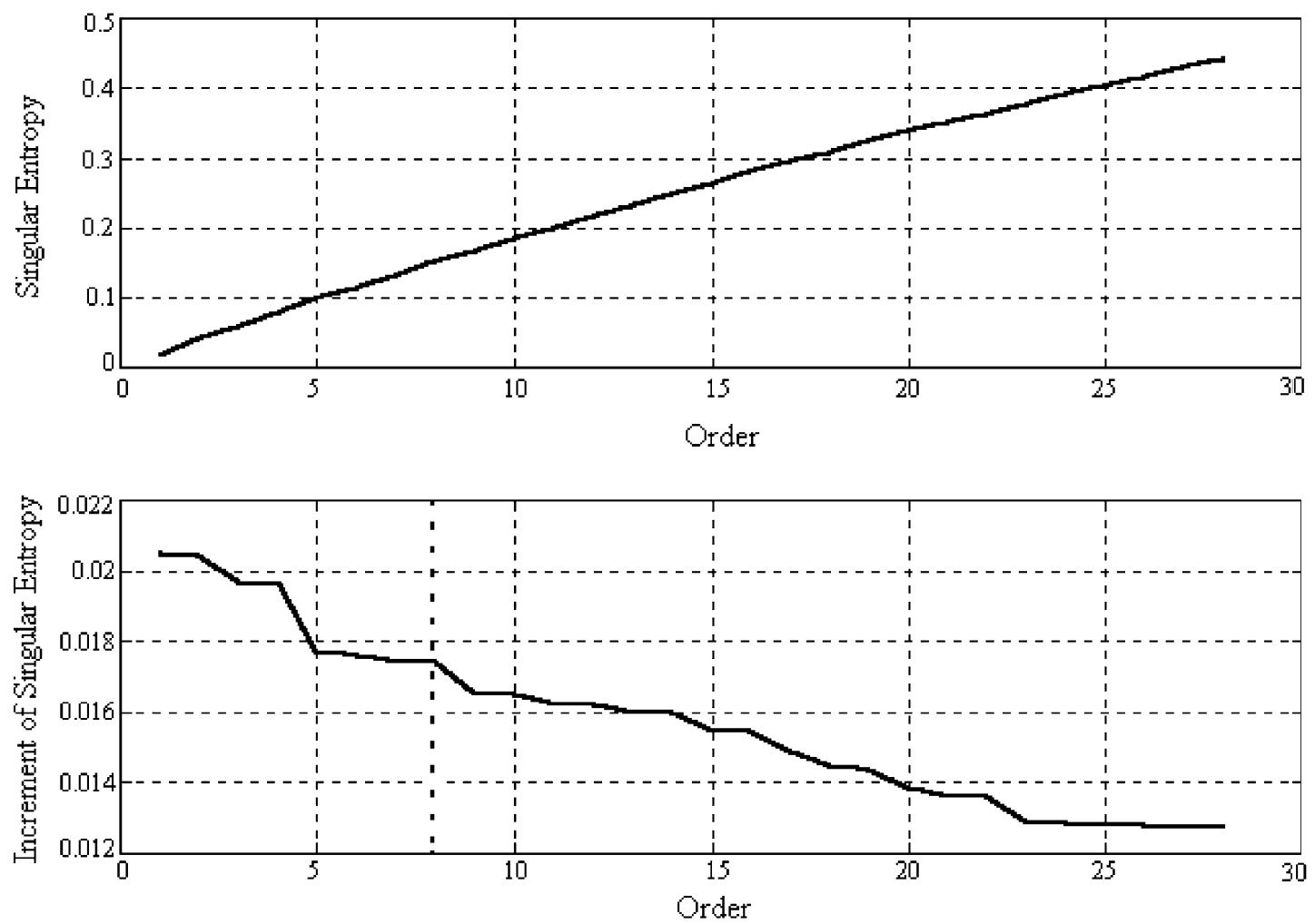

(b) curves of SE and its increment

Fig. 9. Acceleration signal when an outer race failure happened. 

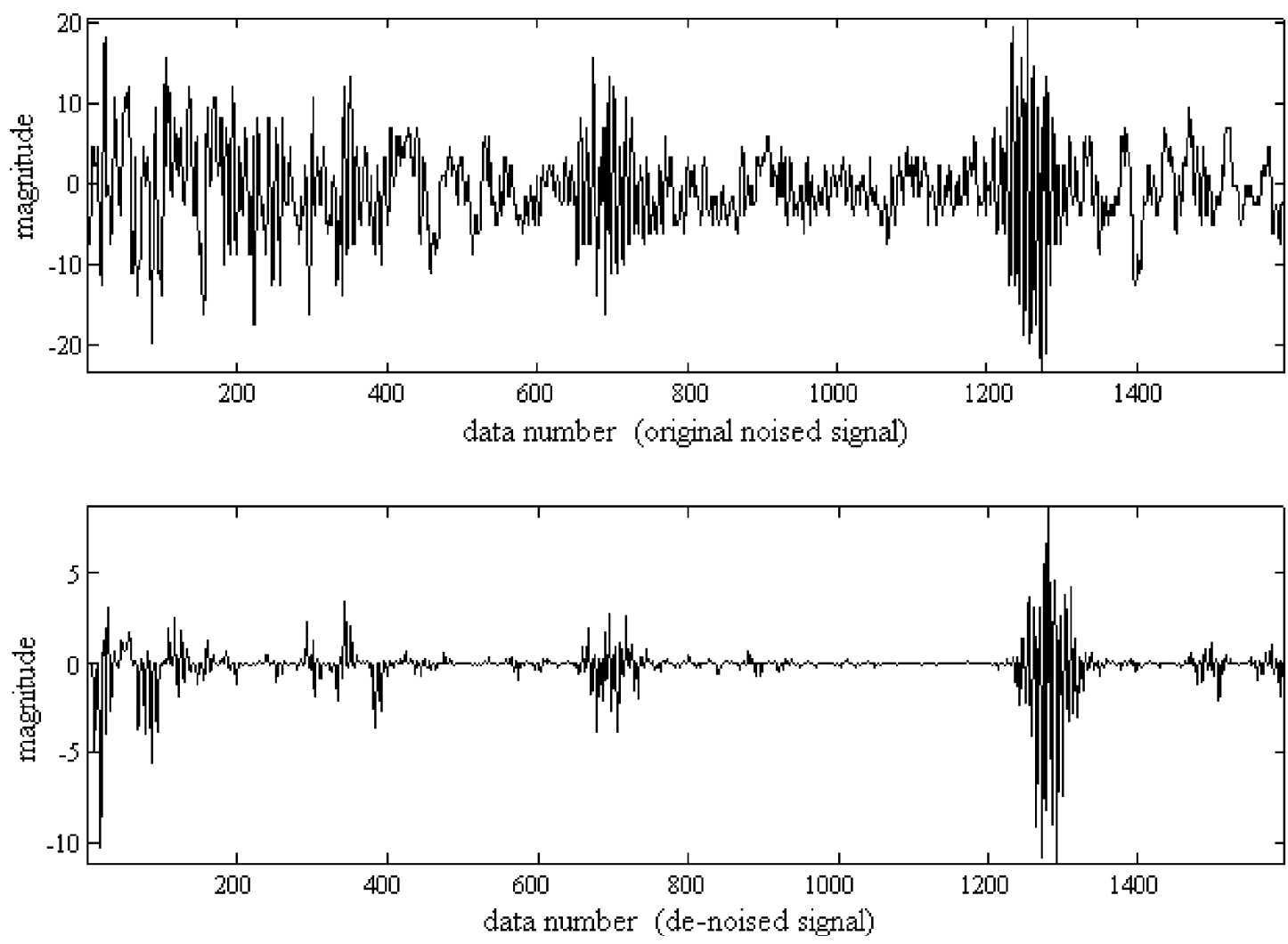

(a) raw and noise-reduced signals
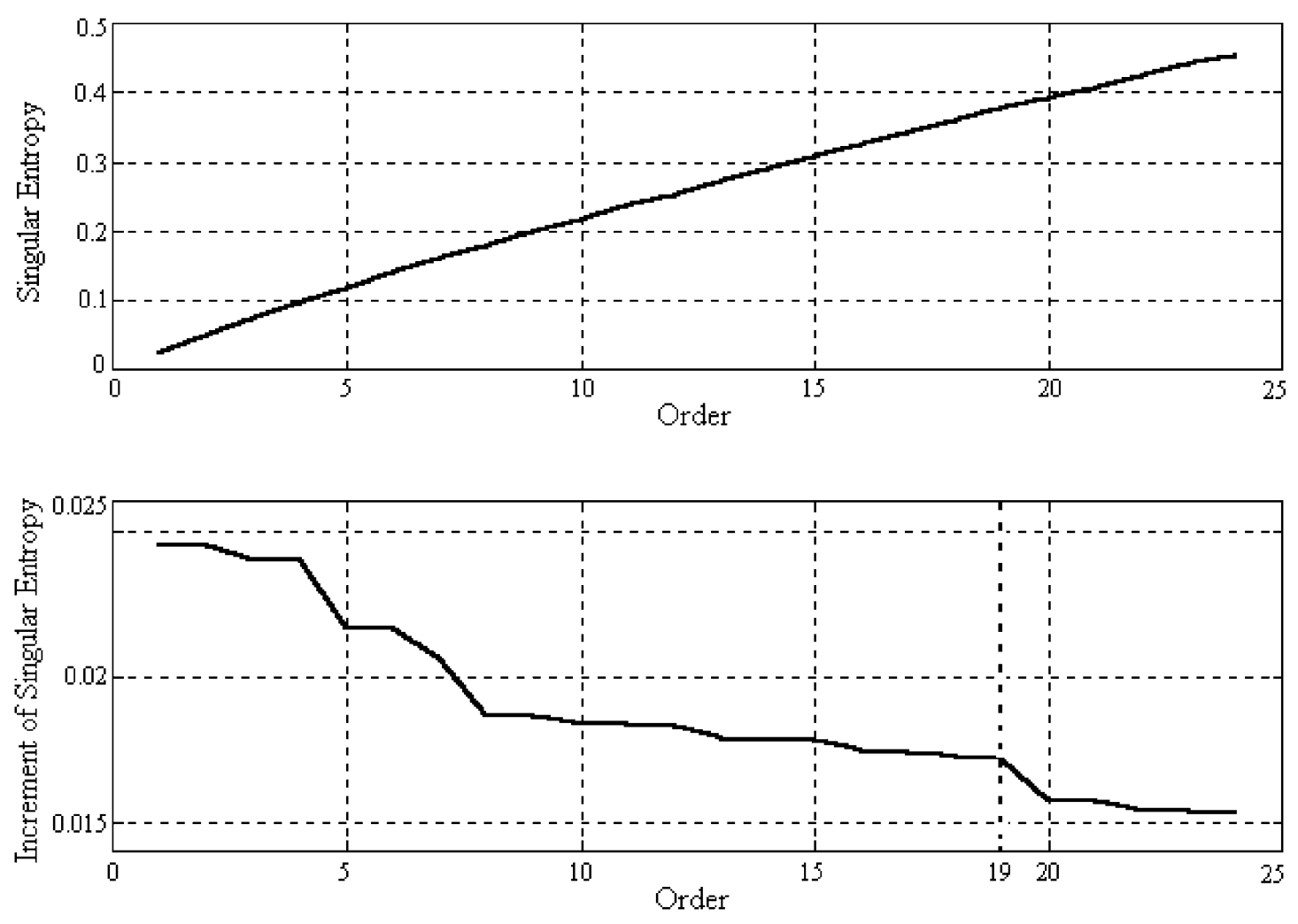

(b) curves of the SE and its increment

Fig. 10. Acceleration signal when a ball failure happened. 


\section{Conclusions and remarks}

In order to develop a more reasonable and practical method for noise reduction, a new concept namely the SE is proposed in this paper. With the aid of the SE, many performances of the SVD-based de-noising method are investigated. Furthermore, a strategy for reasonably determining the optimum de-noising order is developed. Based on this strategy, an advanced SVD-based noise reduction method is proposed. The effectiveness of this advanced method is verified by a series of simulated and practical experiments. From the calculation results and discussions, the following conclusions can be drawn.

1. The determination of the de-noising order is a key link for the SVD-based noise cancellation technique. The saturated order of the SE offers a firm support to this work.

2. The SE of the signals containing same number of frequency components will show the nearly identical variation tendencies.

3. There exists a definite relation between the saturated order of the SE and the number of frequency components contained in the signal, as indicated by Eqs. (7) and (9). Moreover, when there is same number of frequency components contained, the saturated order of the SE of a seriously noise contaminated signal is larger one order than that of a pure signal or a signal that has not been seriously polluted by noise. This is because, the SE of the white noise can reach the saturated state at the first order.

4. The SVD-based de-noising method has an excellent frequency resolution. This guarantees its wonderful noise reduction effect to a great extent.

5. The SVD-based de-noising method has a strong capability. It can still identify the useful information from a noised signal even though the SNR of the signal is very low.

6. With the aid of the SE and its increment $\Delta E$, not only the useful information contained in stationary signals can be easily distinguished, but also the useful information contained in non-stationary signals can be successfully identified.

\section{Acknowledgements}

This research was supported by the National Natural Science Fund of China (ID number: 50205021), the Shaanxi Provincial Natural Science Fund (ID number: 2002E 26 ), and partially supported by the research project of the City University of Hong Kong (ID number: 9040501).

\section{References}

[1] Janacek GJ. Practical time series. London: Arnold; 2001.

[2] Shumway RH, Stoffer DS. Time series analysis and its applications. New York: Springer; 2000.

[3] Kim YY, Hong JC, Lee NY. Frequency response function estimation via a robust wavelet de-noising method. J Sound Vib 2001;244(4): 635-49.

[4] Lin J-W, Laine AF, Bergmann SR. Improving PET-based physiology quantification through methods of wavelet denoising. IEEE Trans Biomed Engng 2001;48(2):202-12.

[5] Shim I, Soraghan JJ, Siew WH. Noise reduction technique for on-line detection and location of partial discharges in high voltage cable. Meas Sci Technol 2000;11(12):1708-13.

[6] Hadjileontiadis LJ, Liatsos CN, Mavrogiannis CC, Rokkas TA, Panas SM. Enhancement of bowel sounds by wavelet-based filtering. IEEE Trans Biomed Engng 2000;47(7):876-86.

[7] Bakhtazad A, Palazoglu A, Romagnoli JA. Process data de-noising using wavelet transform. Intell Data Anal 1999;3(4):267-85.

[8] Presti LL, Olmo G, Bosetto D. Turbo estimation algorithms: general principles, and applications to modal analysis. Signal Process 2000; 80(12):2567-78

[9] Leibrich J, Puder H. A TF distribution for disturbed and undisturbed speech signals and its application to noise reduction. Signal Process 2000;80(9):1761-76.

[10] Jeff L, Rafik G. GMDF for noise reduction and echo cancellation. IEEE Signal Process Lett 2000;7(8):230-2.

[11] Saso T. Residual noise reduction in sign algorithm. IEEE Signal Process Lett 2000;7(8):233-4.

[12] Kuo Chung J, Lin Chien H, Yeh Chia H. Noise reduction of VQ encoded images through anti-gray coding. IEEE Trans Image Process 1999;8(1):33-40.

[13] Windyga, PS. Fast impulsive noise removal. IEEE Trans Image Process 2001;10(1):173-9.

[14] Gharieb RR, Cichocki A. Noise reduction in bra third-or. IEEE Trans Biomed Engng 2001;48(5):501-12.

[15] Muneyasu M, Nishi N, Hinamoto T. A new adaptive center weighted median filter using counter propagation networks. J Franklin Inst 2000;337(5):631-9.

[16] Kitamura K, Iida H, Shidahara M, Miura S, Kanno I. Noise reduction in PET attenuation correction using non-linear Gaussian filters. IEEE Trans Nucl Sci 2000;47(3):994-9.

[17] Sakitani K, Maeda H. Optimum noise reduction of conjugate quadrature filter bank. Signal Process 2000;8(5):819-29.

[18] Laakso TI, Tarczynski A, Paul Murphy N, Valimaki V. Polynomial filtering approach to reconstruction and noise reduction of nonuniformly sampled signals. Signal Process 2000;80(4): $567-75$.

[19] Montgomery SL, Eugene Jr HM. Roundoff noise reduction in cascade realizations of FIR digital filters. IEEE Trans Signal Process 2000; 48(4):1196-200.

[20] Olli V. Minimum-phase FIR filters for delay-constrained noise reduction. IEEE Trans Instrum Meas 1999;48(6):1100-2.

[21] Soon Iy, Koh SN, Yeo CK. Improved noise suppression filter using self-adaptive estimator of probability of speech absence. Signal Process 1999;75(2): 151-9.

[22] Chen Z, mechefske CK. Machine signature identification by analysis of impulse vibration signals. J Sound Vib 2001;244(1):155-67.

[23] Pau JS, Reddy MR, Kumar VJ. A transform domain SVD filter for suppressing of muscle noise artefacts in exercise ECG's. IEEE Trans Biomed Engng 2000;47(5):654-63.

[24] Kazama M, Tohyama M. Estimation of speech components by ACF analysis in a noisy environment. J Sound Vib 2001;241(1):41-52.

[25] Izquierdo MAG, Hernandez MG, Graullera O, Anaya JJ. Signal-tonoise ratio enhancement based on the whitening transformation of colored structural noise. Ultrasonics 2000;38:500-2. 
[26] Yong Hong S, Saratchandram P, Sundararajan N. Minimal resource allocation network for adaptive noise cancellation. Electron Lett 1999;35(9):726-8.

[27] Gialamas TP, Tsahalis DT, Otte D, Van der Auwaraer H, Manolas DA. Substructuring techniques: improvement by means of singular value decomposition (SVD). Appl Acoust 2001; 62(10):1211-9.

[28] Kim WC, Chin IS, Lee KS, Choi J. Analysis and reduced-order design of quadratic criterion-based iterative learning control using singular value decomposition. Comput Chem Engng 2000;24(8):1815-9.

[29] Ray G, Prasad AN, Bhattacharyya TK. Design of decentralized robust load-frequency controller based on SVD method. Comput Electr Engng 1999;25(6):477-92.

[30] Street AM, Lukama L, Edwards DJ. Radio imaging using SVD prony. Electron Lett 2000;36(13):1150-2.

[31] Vidya V, Aravena Jorge L. Nonstationary signal classification using pseudo power signatures: the matrix SVD approach. IEEE Trans
Circuits Syst II: Analog Digital Signal Process 1999;46(12): 1497-505.

[32] Dale G, David B. Feature sets for nonstationary signals derived from moments of the singular value decomposition of Cohen-Posch (positive time-frequency) distributions. IEEE Trans Signal Process 2000;48(5):1498-503.

[33] Knockaert L, De Backer B, De Zutter D. SVD compression, unitary transforms, and computational complexity. IEEE Trans Signal Process 1999;47(10):2724-9.

[34] Kuo SR, Yeih W, Wu YC. Applications of the generalized singularvalue decomposition method on the eigenproblem using the incomplete boundary element formulation. J Sound Vib 2000; 235(5):813-45.

[35] Chen JT, Huang CX, Wong FC. Determination of spurious eigenvalue and multiplicities of true eigenvalues in the dual multiple reciprocity method using the singular-value decomposition technique. J Sound Vib 2000;230(2):203-19. 